\title{
The X-linked inhibitor of apoptosis (XIAP) prevents cell death in axotomized CNS neurons in vivo
}

\author{
S Kügler ${ }^{\star, 1,3}$, G Straten ${ }^{1,3}$, F Kreppel ${ }^{1}$, S Isenmann ${ }^{1}$, P Liston ${ }^{2}$ \\ and M Bähr ${ }^{1}$ \\ ${ }^{1}$ Department of Neurology, University of Tuebingen, Medical School \\ Verfuegungsgebaeude, Auf der Morgenstelle 15, 72076 Tuebingen, Germany \\ 2 Molecular Genetics Research Laboratory, Children's Hospital, and Apoptogen \\ Inc., Ottawa, Canada \\ ${ }^{3}$ The first two authors contributed equally to this work \\ * Corresponding author: S Kügler, Department of Neurology, University of \\ Tuebingen, Medical School Verfuegungsgebaeude, Auf der Morgenstelle 15, \\ 72076 Tuebingen, Germany. Tel: +7071 29 87615; Fax: +7071 29 5742; \\ E-mail: kuegler@uni.tuebingen.de
}

Received 13.10.99; revised 10.3.00; accepted 4.5.00

Edited by FD Miller

\begin{abstract}
The inhibition of neuronal apoptosis in acute traumatic and ischemic injuries as well as in long term neurodegenerative disorders like spinal muscular atrophy and possibly Alzheimer's disease is a fundamental requirement for a therapeutic strategy. In this study we used an established in vivo model system of induction of neuronal apoptosis in the CNS to evaluate the properties of the X-linked inhibitor of apoptosis protein (XIAP) to inhibit secondary cell death after axonal lesions. We used adenoviral vectors to transduce retinal ganglion cells after axotomy of the optic nerve of adult rats. Vector application was performed at the optic nerve stump so that only the lesioned retinal neurons could be transduced. We found XIAP to be as effective as the viral broad spectrum caspase inhibitor protein p35. These findings suggest that axotomized RGCs degenerate through class II caspase activity and furthermore offer the possibility of using mammalian XIAP protein to inhibit neuronal apoptosis as a basis for a regenerative therapy in the CNS. Cell Death and Differentiation (2000) 7, 815-824.
\end{abstract}

Keywords: apoptosis; CNS; retina; XIAP; adenovirus

Abbreviations: DISC, death inducing signaling complex; IAP, inhibitor of apoptosis protein; Flip, FLICE-inhibitory protein; RGC, retinal ganglion cell; CNTF, ciliary neurotrophic factor; ON, optic nerve

\section{Introduction}

Although apoptosis is an important physiological process in the development of the central nervous system ${ }^{1,2}$ it has detrimental effects in the finally differentiated CNS, because degenerated neurons cannot be replaced in higher vertebrates. Apoptotic neuronal cell death has been claimed to contribute to neurodegenerative disorders such as spinal muscular atrophy, ${ }^{3}$ and Alzheimer's disease ${ }^{4-6}$ and is also responsible for cell loss after ischemia and traumatic injury. ${ }^{7-10}$ It is of great clinical importance to reduce the neuronal cell loss as much as possible, allowing both for compensatory activity and as a basis for regenerative therapy.

The apoptotic program is executed by a family of specialized proteases termed caspases. ${ }^{11,12}$ The activation of inactive zymogens and the activity of the caspases can be inhibited by proteins expressed either by viruses or endogenously in mammalian cells. Viral anti-apoptotic proteins include p35 and IAP from baculovirus, ${ }^{13,14} \mathrm{CrmA}$ from cowpoxvirus ${ }^{15}$ and the $v$-Flip proteins from herpes viruses. $^{16}$ Several mammalian homologs of these viral proteins have been identified. The mammalian IAP homologs include XIAP, NAIP, cIAP 1 and 2 and survivin; ${ }^{17-20}$ a cellular Flip homolog with different isoforms has also been identified. ${ }^{21}$

The various anti-apoptotic proteins exhibit different caspase-inhibitory properties. While CrmA and the Flips target the DISC-complex and inhibit caspase 8 activation, ${ }^{15,21}$ the IAPs directly inhibit the effector caspases 3 and 7 , the BIR3-Ring domains of XIAP have also inhibitory properties for caspase $9 .{ }^{17,19,22}$ p35 is a broad spectrum caspase inhibitor with similar $\mathrm{K}_{\mathrm{i}}$ for all caspases tested. ${ }^{13}$

An established experimental paradigm for the induction as well as for studying the subsequent inhibition of neuronal apoptosis in the CNS, is the lesion of the retinotectal projection in the rat. Axotomy of the rat optic nerve leads to apoptotic cell death of the retinal ganglion cells (RGCs), which are the only retinal neurons projecting into the optic nerve. Fourteen days post lesion, over $80 \%$ of the retinal ganglion cells have degenerated. ${ }^{23-27}$ RGCs can be efficiently pre-labeled by retrogradely transported fluorescent dyes and thus cell survival can be quantitatively determined by counting labeled cells in the whole-mount retina. Also RGCs can be transduced by retrogradely transported viral vectors such as recombinant adenoviruses, ${ }^{28,29}$ when applied at the ocular side of the lesioned optic nerve stump.

It is the main advantage of this experimental system that only those neurons which are directly affected by the lesion can be transduced by the vectors. In this experimental paradigm p35 but not CrmA has been shown to promote RGC survival over a 14 day period post-lesion, indicating that cell death of axotomized ganglion cells is not death receptor dependent. ${ }^{30}$ To further investigate the mechanisms leading to cell death in axotomized neurons and to evaluate the possibility of using autologous, cellular antiapoptotic proteins for protecting neurons from apoptosis in this paradigm, we used an adenoviral vector coding for $\mathrm{XIAP}$, a specific class II (caspases 3 and $7^{19}$ ) caspase inhibitor to transduce axotomized retinal ganglion cells.

We show that Ad.XIAP transduction is as efficient in inhibiting apoptosis in axotomized neurons as is Ad.p35 transduction, whereas two control vectors exerted no 
protective effect. These results indicate that viral p35 may be replaced in studies of inhibition of apoptosis or in therapies by the cellular XIAP with identical efficacy. These findings might be particularly important for long term studies in the CNS such as in the treatment of slowly progressing neurodegenerative disorders. Also, we add further evidence to recent findings ${ }^{9,27}$ that class II caspases are major executioners of apoptosis in lesioned CNS neurons.

\section{Results}

\section{Vectors and vector application}

We have used the Ad.Xiap vector along with several control vectors. Negative controls consisted of (i) vehicle alone (PBS $/ 10 \%$ glycerol), (ii) a vector without a transgene in the $E 1$ region (Ad.dE1) or (iii) a vector coding for EGFP in E1 (Ad.GFP). As a positive control, a vector coding for p35 (Ad.p35) was used, since it has already been shown to be neuroprotective in this experimental paradigm. ${ }^{30}$ The Ad.Xiap vector was tested in vitro by transduction of cerebellar granule neurons after potassium withdrawal and showed inhibition of caspase 3 activity and enhancement of cell survival (Gerhard E, Kügler S, Leist $M$, Bähr $M$, Nicotera $P$, Schulz JB, manuscript in preparation). Furthermore, we investigated the neuroprotective properties of a vector coding for a secreted form of the neurotrophin CNTF (Ad.CNTF). The adenoviral vectors used in this study are schematically illustrated in Figure 1. Viral particles were applied to the ocular side of the lesioned optic nerve stump in a $5 \mu \mathrm{l}$ droplet; the viscosity of the solution (PBS/10\% glycerol) kept the suspension in the open dura. The titre of all of the vectors was 2.5$3 \times 10^{8}$ p.f.u. $/ \mu$ l. The viral suspension was not removed before closing the wound.

\section{Monitoring of transgene expression in the retina}

In order to demonstrate that RGCs are the only retinal cells transduced by the adenoviral vector application at the nerve stump and to allow for a semiquantitative determination of the rescue effects obtained after the various vector applications, we used an Ad.lacZ vector as reporter. In cryosections from transduced retinae, we found cells positive after staining with an anti- $\beta$ Gal antibody in the ganglion cell layer only (Figure $2 \mathrm{~A}$ ), while no stained cells were detected in the other retinal layers or in retinae which were not transduced (Figure 2B). We also detected $\beta$ galactosidase expression in extraretinal tissue, as shown after histochemical X-Gal staining of retinal sections. Figure $2 \mathrm{C}$ shows transduced cells in the nerve stump, in the dura mater and in non-neural connective tissue. However, in retinae prepared for counting of surviving RGCs and for RNA extractions, these extraretinal tissues are virtually completely removed (Figure 2D). Part (a) of Figure 2D shows a whole mounted retina (histochemically stained with $\mathrm{X}$-Gal for $\beta$-galactosidase activity) with labeled cells exclusively in the uppermost ganglion cell layer. Preparations of retinae for counting surviving RGCs and for RNA extraction were carried out in the same manner as for the $\beta$ galactosidase detection shown, thereby ensuring that no other transduced cells than RGCs were present in the preparations. Such specimen showed a distribution of labeled cells identical to that obtained after vector application to the superior colliculus (Figure 2D part b). This brain region is the target area for the axons of the RGCs. Obviously, only RGCs can be transduced by retrograde transport of the adenoviral vector after stereotaxic injections into the superior colliculus. Since the optic layer of the superior colliculus is a very thin but relatively large area which also shows topographically ordered projections of RGC axons, we found that in about $70 \%$ of the animals injected into the superior colliculus only part of

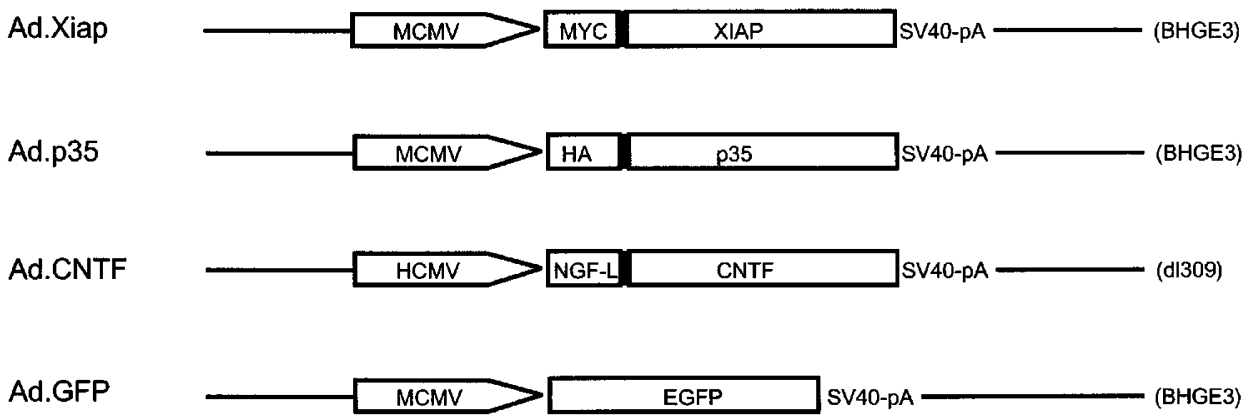

Ad.dE1

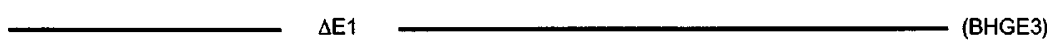

Ad.lacZ

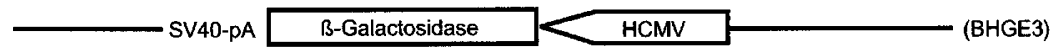

Figure 1 Schematical representation of the adenoviral vectors used in this study. The transgenic E1 regions are shown in detail. MCMV=murine cytomegalovirus promoter; $\mathrm{HCMV}=$ human cytomegalovirus promoter; SV40pA=SV40 polyadenylation site; $M Y C=c-m y c$ epitope; $H A=H A$ epitope; $N G F-L=$ nerve growth factor leader sequence; BHGE3, dl309=adenoviral backbones 


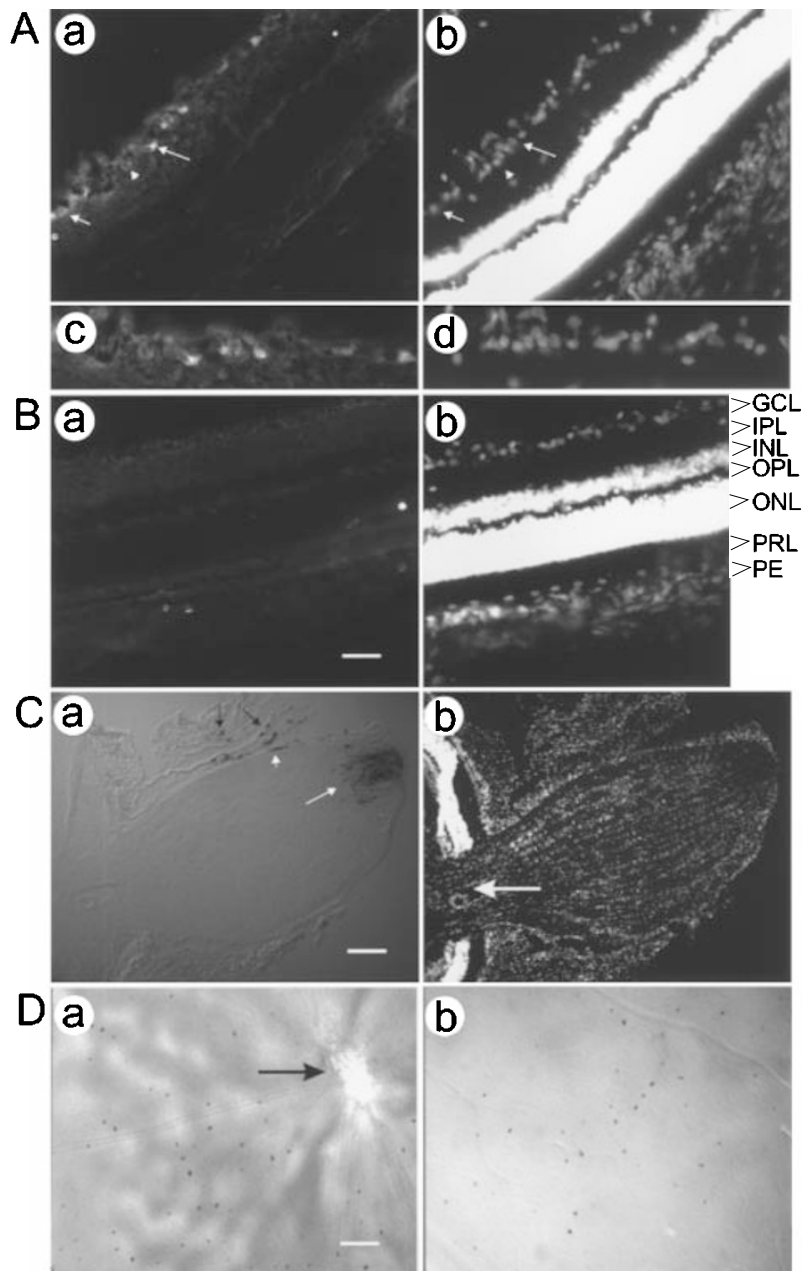

Figure 2 Transduction of the retina by the Ad.lacZ reporter vector. (A) Cryosection of a retina transduced via the nerve stump by the Ad.lacZ vector and stained with an anti $\beta$-galactosidase antibody (a). As revealed by DAPI staining of the same section (b), labeled cells were present in the ganglion cell layer only. Cells with different staining intensities were detected, as shown by arrows (long arrow=strongest signal, arrowhead=weakest signal). Enlargements of the GCL are shown in $c$ (anti $\beta$-galactosidase stain) and $\mathrm{d}$ (DAPI stain). (B) Control retina, without axotomy/vector application. (a) anti $\beta$ galactosidase antibody, (b) DAPI stain. In (b) the retinal layers are described: $\mathrm{GCL}=$ ganglion cell layer, IPL=inner plexiform layer, INL=inner nuclear layer, $\mathrm{OPL}=$ outer plexiform layer, $\mathrm{ONL}=$ outer nuclear layer, $\mathrm{PRL}=$ photoreceptor layer, PE=pigment epithelium. (C) Vector (Ad.lacZ) application at the optic nerve stump results in transduction of extraretinal tissue as shown by histochemical (X-Gal) staining of retinal sections and the nerve stump. $\beta$ galactosidase expression is detected in the nerve stump (white arrow), in the dura mater (white arrowhead) and in non-neural connective tissue (black arrows). (a) X-Gal stain, (b) DAPI stain, the large white arrow marks the optic disc. (D) Whole mounted retinae, histochemically stained with X-Gal for $\beta$ galactosidase activity. (a) Ad.lacZ transduction via the nerve stump, (b) Ad.lacZ transduction by vector injection into the contralateral superior colliculus. The black arrow in (a) points to the position of the optic disc, which is removed from the specimen together with the other extraretinal tissue during the preparation. Scale bar $=100 \mu \mathrm{m}$ in $\mathbf{A}$ and $\mathbf{B}, 350 \mu \mathrm{m}$ in $\mathbf{C}$ and $450 \mu \mathrm{m}$ in D

the retinae were transduced. Therefore, application of the vectors directly to the nerve stump is a favorable way of delivering transgenes specifically to RGCs in the retina.
After Ad.lacZ application, we detected $\beta$-galactosidase in about $10-20 \%$ of all cells in the ganglion cell layer as determined by counting both $\beta$-galactosidase positive cells and DAPI stained nuclei. Since about $50 \%$ of all cells in the ganglion cell layer are displaced amacrine cells (which do not project to the optic nerve), we estimate that we transduced about $20-40 \%$ of the total retinal ganglion cell population by this approach.

Since RGCs are the only retinal cells transduced by the vector application in our experimental setup, transgene expression could be readily monitored by RT-PCR of RNA extracted from isolated retinae. We investigated transgene expression from the Ad.Xiap, Ad.CNTF and Ad.p35 vectors at 7 and 14 days after axotomy and transduction. RNA was reverse-transcribed with primers specific for the respective transgene mRNAs plus a primer for the internal standard, G3PDH. To exclude the possibility of amplification of endogenous Xiap or CNTF mRNA, the sense primers for the virally encoded transgenes were placed in the $\mathrm{N}$ terminal fusions of these peptides (i.e. the Myc tag in the XIAP and the NGF leader sequence in the CNTF transgene).

For all three transgenes we used a nested PCR approach for highest specificity. Under the amplification conditions applied no products were detectable after the first amplification, whereas correctly-sized products were obtained after the second, nested PCR amplification (Figure 3A,C,D). Densitometric scanning of the obtained PCR products and comparison of expression levels at 7 and 14 days post axotomy/transduction, respectively, revealed a moderate decrease in all three transgene mRNA levels as compared to the G3PDH standard. Depending on the individual experiment, the relative expression levels after 14 days were $45-80 \%$ as compared to the levels at 7 days ( $n=3$ amplifications for each cDNA). No amplification products were obtained in the reverse transcriptase negative reactions, thus excluding the potential amplification of vector genomes.

The XIAP protein translated from the respective Ad vector was $\mathrm{N}$-terminally tagged with a c-myc epitope. This epitope allows the detection of the fusion protein without using a XIAP specific antibody. In protein extracts from Ad.Xiap-transduced MRC-5 fibroblasts, the myc-tagged XIAP was readily detected by using the anti-myc antibody 9E10 (Figure 3B). No protein was detected from control transduced cells (data not shown). However, in extracts from Ad.Xiap transduced retinae, we were not able to detect the myc-tagged XIAP protein but only detected a protein migrating at the size of endogenous c-Myc. Although the size difference between myc-tagged XIAP and $\mathrm{C}-\mathrm{Myc}$ as predicted from the amino acid sequence is only small (67 versus $66 \mathrm{kDa}$ ), both proteins were readily separated on PAA gels. As shown in Figure 3B, the myctagged XIAP from lysates of MRC-5 cells migrates somewhat above the band detected in untransduced retinae. In Ad.Xiap transduced retinae, no additional band is detected, indicating that the amount of XIAP produced by the transduced RGCs is not sufficient for detection by Western blotting, most likely because RGCs make up only a very small percentage (below 1\%) of all retinal cells. 
According to the results obtained by Western blotting, the anti-myc-tag antibody stained retinal ganglion cells and cells of the inner and outer nuclear layers by immunohistochemistry, both in axotomized controls and in axotomized
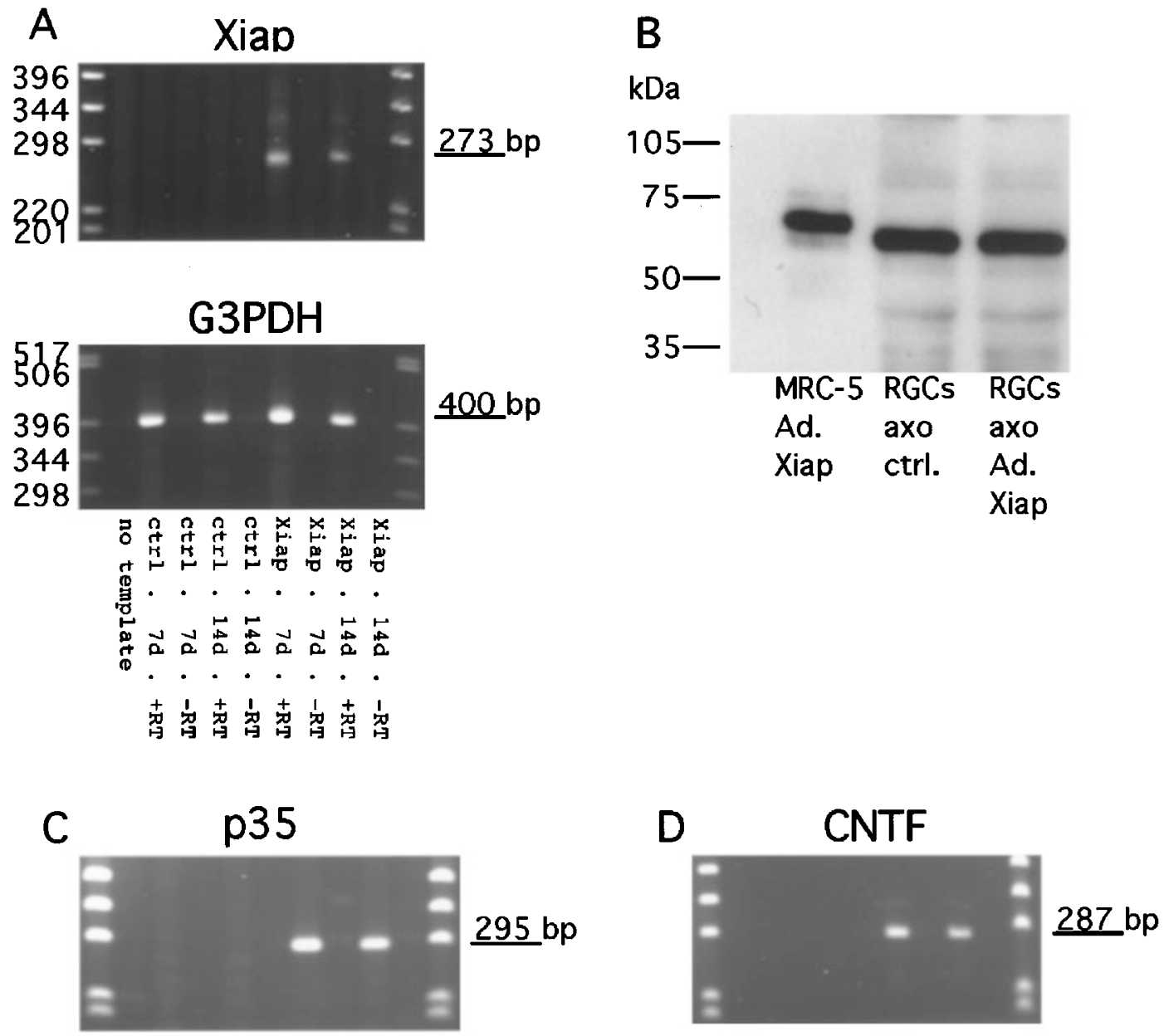

\section{G3PDH}

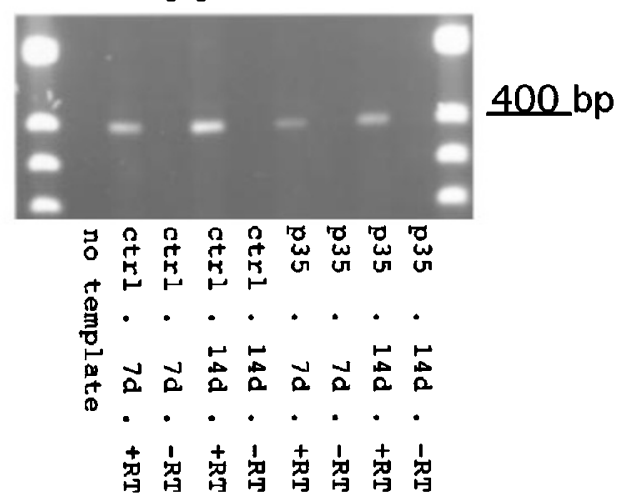

\section{G3PDH}

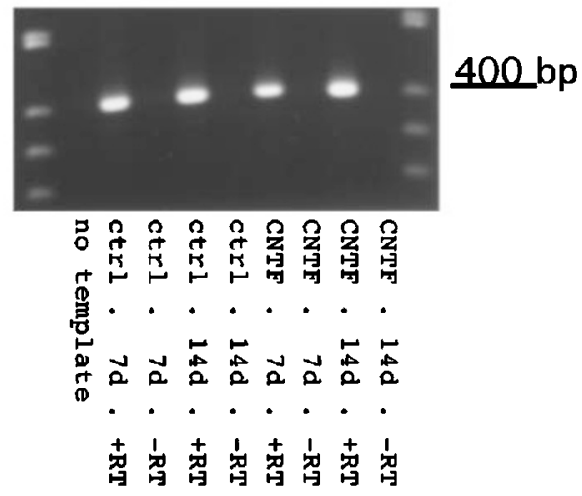

Figure 3 Amplification products obtained by nested RT-PCR from retinae transduced either with Ad.Xiap (A), Ad.p35 (C) or Ad.CNTF (D). The upper ethidium bromide stained gel shows the PCR products specific for the mRNAs transcribed from Ad.Xiap, Ad.p35 and Ad.CNTF, respectively. The lower gel shows the corresponding G3PDH mRNA specific PCR products. The size of the molecular weight markers is indicated on the left in (A), the predicted size of the respective amplification products is indicated on the right side of each gel. ctrl.=RNA extracted from non-transduced retinae (from the respective contralateral eyes); Xiap, p35, CNTF=RNA extracted from viraly transduced retinae; $7 \mathrm{~d}=$ RNA extracted 7 days after axotomy/transduction; 14d=RNA extracted 14 days after axotomy/ transduction; +RT=reverse transcription reaction with reverse transcriptase; $-\mathrm{RT}=$ reverse transcription reaction without reverse transcriptase. (B) Western blot analysis using the $9 \mathrm{E} 10$ anti-myc antibody. Protein extracts from Ad.Xiap transduced MRC-5 fibroblasts (MRC-5 Ad.Xiap), axotomized but not transduced retinae (RGCs axo ctrl.) and axotomized/Ad.Xiap transduced retinae (RGCs axo Ad.Xiap) were probed with the 9E10 anti myc antibody. Molecular weight markers are indicated on the left. A representative gel of $n=5$ experiments is shown 
and Ad.Xiap transduced retinae (not shown). Therefore, transduction with the Ad.lacZ reporter vector had to be used to determine the amount of RGCs transducable by the vector administration at the nerve stump.

\section{Determination of the number of surviving RGCs after axotomy and transduction with viral vectors}

To allow for quantification of surviving RGCs after ON axotomy and various treatments, RGCs were fluorescently prelabeled by injection of the retrogradely transported fluorescent dye, Di-I, into the superior colliculi (the target region of the RGCs in the midbrain) of neonatal rats. Axotomy and subsequent vector application were performed on these animals when they were 10 weeks old.

Labeled RGCs were counted in whole-mounted retinae 14 days after the axotomy and the viral vector applications. The membrane bound Di-I fluorophor showed a characteristic punctate staining pattern in RGCs of unlesioned control retinae (Figure 4A). For quantification of surviving RGCs, only those cells were counted which had a normal morphology as compared to unlesioned RGCs and which have retained the uniformly distributed label (marked $\mathrm{C}=$ counted in Figure 4B). Cells with obviously condensed membranes as judged from strong spotlike accumulation of label (marked $\mathrm{N}=$ not counted in Figure 4B) were excluded from counting. Cells which showed only limited label accumulation were counted as surviving cells since focusing revealed a normal morphology and such partial label accumulation was also observed in RGCs from control retinae.

In the absence of a treatment or after vehicle application approximately $320 \mathrm{RGCs} / \mathrm{mm}^{2}$ showed normal morphology and homogenous fluorescence which was well in accordance with previous studies using other fluorescent labels. After transduction with Ad. Xiap, about $650 \mathrm{RGCs} / \mathrm{mm}^{2}$ survived axotomy for the 14 days period postlesion. This means that about $330 \mathrm{RGCs} / \mathrm{mm}^{2}$ have been rescued from cell death due to transduction with this vector. Accordingly, about $380 \mathrm{RGCs} / \mathrm{mm}^{2}$ and about $340 \mathrm{RGCs} / \mathrm{mm}^{2}$ were
A

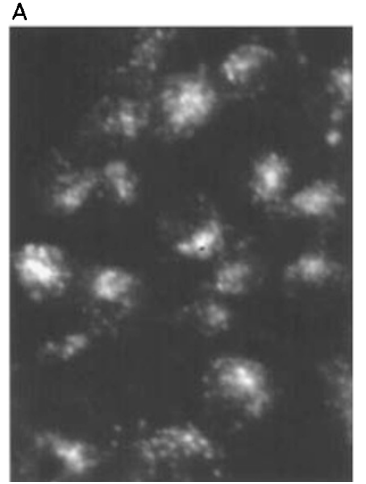

B

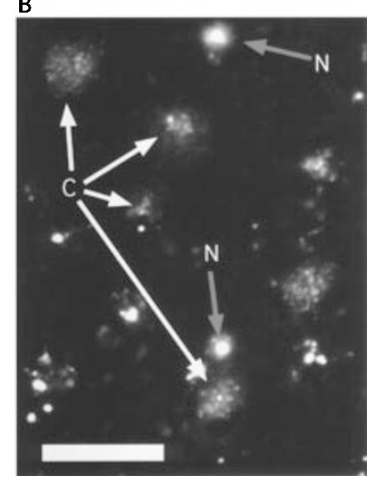

Figure 4 Fluorescent photographs of flat mounted retinae. Di-l labeled retinal ganglion cells in either untreated (no axotomy) control retina $(\mathbf{A})$ or axotomized and Ad.Xiap transduced retina (B) are shown. $(\mathbf{C})$ and white arrows denote RGCs which were counted as surviving cells, $\mathrm{N}$ and gray arrows denote labeled cells excluded from counting. Scale bar $=50 \mu \mathrm{m}$ rescued by the Ad.CNTF and the Ad.p35 vector transduction, respectively. As compared to the control (null) vector Ad.dE1, which did not code for a transgene, we found significant rescue effects for the Ad.Xiap vector $(P=0.006)$, the Ad.CNTF vector $(P=0.014)$ and the Ad.p35 vector $(P=0.009)$. No statistically significant differences in the rescue rates of the Ad.XIAP, Ad.p35 and Ad.CNTF vectors were observed. The GFP coding vector exerted no significant rescue effect as compared to the vehicle control or the Ad.dE1 null vector. Figure 5 shows the numbers of surviving RGCs counted 14 days post axotomy and viral transductions. The relative amount of RGCs surviving axotomy was determined as follows: in normal, untreated control retinae about $1900 \mathrm{RGCs} / \mathrm{mm}^{2}$ were counted, which is well in agreement with previous studies. ${ }^{23,26,27}$ Transduction with the Ad.lacZ reporter vector revealed that about $30 \pm 10 \%$ of the total population of RGCs is transduced by our vector application mode, which corresponds to $570 \pm 190 \mathrm{RGCs} / \mathrm{mm}^{2}$. Since after Ad.Xiap application $330 \mathrm{RGCs} / \mathrm{mm}^{2}$ were still alive 14 days post axotomy due to the transduction with this vector, we estimate that roughly half of the transduced RGCs were rescued from apoptosis by this approach. Note that both histochemistry of whole mounted retinae (X-Gal staining) and immunological detection of $\beta$-galactosidase expression on frozen sections revealed pronounced differences in the signal intensities, suggesting differences in the uptake of the amount of vector by individual axons, which seems to result in quite different amounts of transgene production.

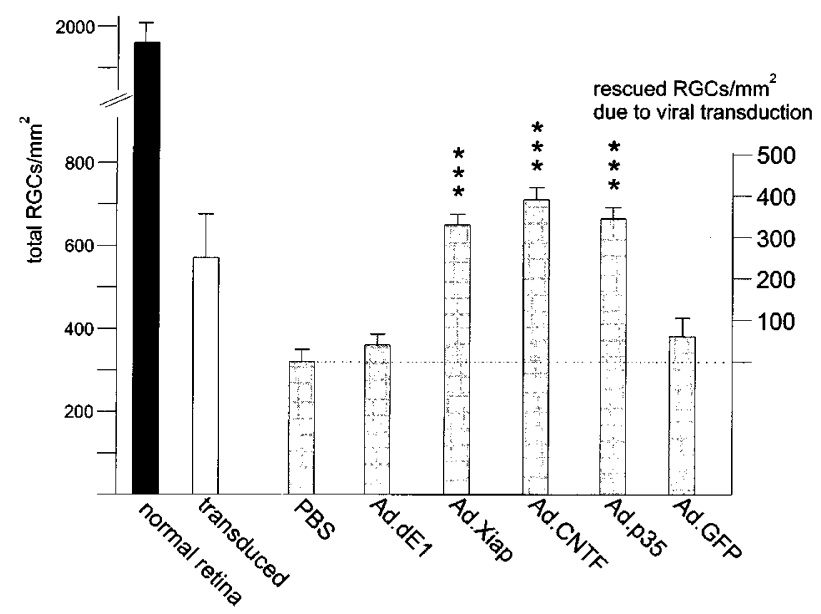

Figure 5 Quantitative representation of RGCs in control and vector transduced retinae. Left scale: total number of $\mathrm{RGCs} / \mathrm{mm}^{2}$ counted 14 days post lesion and vector administration or in controls; right scale: number of surviving RGCs $/ \mathrm{mm}^{2}$ subtracted by the number of RGCs surviving after vehicle application. The dashed horizontal line represents 'zero' for this scale. The total number of RGCs in retinae of control animals (no axotomy) is shown by the black bar ('normal retina'). The number of RGCs estimated to be transduced by application of the reporter vector Ad.lacZ to the sectioned nerve stump is shown by the white bar ('transduced'). Gray shaded bars represent the number of RGCs surviving axotomy 14 days postlesion after the indicated vector applications. $n=5$ for vehicle (PBS $/ 10 \%$ glycerol), $n=5$ for Ad.dE1 (null) vector, $n=6$ for the Ad.Xiap vector, $n=4$ for the Ad.CNTF vector, $n=5$ for the Ad.p35 vector and $n=3$ for the Ad.GFP vector. Values are shown as mean \pm S.E.M. ${ }^{* * *}$ Denotes statistical significant difference to the Ad.dE1 null vector application (Ad.Xiap $P=0.006$, Ad.CNTF $P=0.014$, Ad.p35 $P=0.009$ ) 


\section{Endogenous XIAP expression in the rat retina}

As shown above, about $18 \%$ of the total RGC population survived axotomy without any treatment. It is unknown by which means these cells are protected from apoptosis. By staining cryosections from control retinae with an anti-XIAP antibody, we detected strong XIAP immunoreactivity in the ganglion cell layer and weaker immunoreactivity in the inner nuclear layer (Figure 6B). Specificity of the antibody was confirmed by omitting it from the labeling reaction and by mono-specificity on Western blots. Furthermore, RT-PCR with subsequent restriction digest analysis of the PCR fragments obtained for two XIAP specific amplicons revealed expression of endogenous XIAP mRNA in the retina (data not shown).

The RGCs of the respective animals were prelabeled by fluorogold injection into the superior colliculi, allowing to precisely discriminate between RGCs and e.g. displaced amacrine cells in the retina. As judged from whole mounted preparations of such retinae, the labeling efficiency of RGCs is about $95 \%$ (not shown). A comparison between the staining patterns obtained by either the anti-XIAP antibody or the fluorogold label revealed that in the

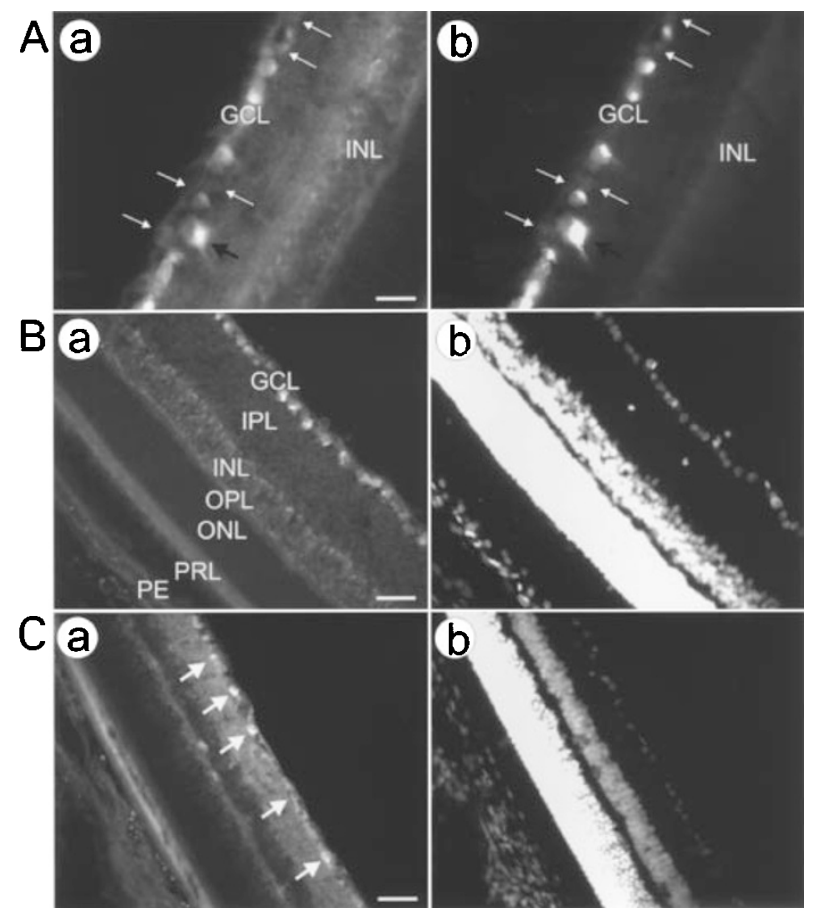

Figure 6 XIAP expression in the retina. (A) Cryosection of a normal control retina from an untreated animal, showing XIAP immunoreactivity (a) and RGCs retrogradely labeled by fluorogold injection into the superior colliculus (b). White arrows point to displaced amacrine cells, which are neither labeled by fluorogold nor by the anti XIAP antibody. The black arrow points to a retina ganglion cell which belongs to the subpopulation of large RGCs, showing the strongest XIAP immunoreactivity. (B) Cryosection of a normal control retina, stained with the anti XIAP antibody (a) and DAPI (b). Strong XIAP immunoreactivity is seen in many cells of the ganglion cell layer while weak immunoreactivity is detected in the inner nuclear layer. (C) Cryosection of a retina prepared 14 days post axotomy and Ad.XIAP application at the nerve stump. Anti XIAP antibody stain (a) and DAPI stain (b). Arrows point to RGCs still alive 14 days postlesion which show substantial XIAP immunoreactivity ganglion cell layer XIAP immunoreactivity is present nearly exclusively in RGCs (Figure 6A). We did not detect XIAP immunoreactivity in displaced amacrine cells in the ganglion cell layer, in cells with microglial nuclear phenotype, in the outer nuclear layer, in the photoreceptor layer nor in the main glial fraction of the retina, the Muller glia cells.

XIAP immunoreactivity was also detected in retinae from animal subjected to axotomy and Ad.XIAP application 14 days postlesion, with a clearly reduced number of immunoreactive RGCs as compared to unlesioned controls (Figure 6C).

\section{Discussion}

Apoptotic cell death contributes to neuronal degeneration in several paradigms including traumatic injury, ischemia/ stroke, spinal muscular atrophy and probably Alzheimer's disease. ${ }^{3-8,31}$ The prevention of neuronal degeneration is a fundamental basis for regenerative therapy and is also important in allowing compensatory remodeling of neuronal connections. We have recently shown that the baculovirus protein p35, which is a broad spectrum caspase inhibitor, efficiently prevents neuronal cell death in an in vivo model of axonal injury, the axotomy of the rat optic nerve. ${ }^{30}$ In contrast, CrmA, which is a potent inhibitor of caspases 1 and 8 , showed no neuroprotective effects in this experimental paradigm.

We have now attempted to substitute the viral p35 by an autologous mammalian protein, X-linked inhibitor of apoptosis (XIAP). XIAP, C-IAP-1 and C-IAP-2 have been shown to directly inhibit the downstream caspases 3 and 7 but not caspases 1, 6, 8 and 10..$^{10,17,19,32}$ XIAP was chosen for this study because it showed substantially higher caspase inhibitory activity than C-IAP-1 and $-2 .^{19}$

Our results demonstrate that transduction with Ad.Xiap was as efficient in preventing ganglion cell death after axotomy as transduction with Ad.p35. Since XIAP is a specific type II caspase inhibitor, this finding adds further evidence to recent results demonstrating that caspase 3 is a major executioner of apoptosis in axotomized neurons. ${ }^{27}$ However, recent findings suggest that IAPs may possess non caspase dependant anti apoptotic properties through their capability to interfere with the TNF receptor mediated NF-kB activation. ${ }^{33,34}$ In our group, we have investigated whether NF-kB is translocated to the nucleus following axotomy by both gel-shift assays and immunocytochemistry and have never observed such a translocation (R Diem and B Bähr, unpublished observations). Therefore, in agreement with previous experiments employing synthetic caspase inhibitors in the axotomy model, ${ }^{27}$ we would suggest that the Xiap mediated anti-apoptotic mechanism in axotomized RGCs depends on class 2 caspase inhibition.

It has recently been reported that Xiap overexpression was neuroprotective in hippocampal CA1 neurons after transient ischemia. ${ }^{9}$ In this case the vector was applied 7 days before the lesion (four vessel occlusion). In the present work, we applied the vector directly after lesioning which still resulted in highly significant neuroprotection. As the viral vector must be retrogradely transported after transduction, enter the nucleus and be unpacked before transcription of the transgene can begin, it appears to be 
sufficient to express the protective protein in the neuron some hours post-lesion. However, it remains to be determined whether there is a therapeutic window for the delivery of different anti-apoptotic proteins in the different experimental paradigms of neuronal apoptosis.

At present, however, it appears that XIAP is an efficient autologous factor for the inhibition of neuronal apoptosis in the CNS. For long term expression of protective proteins which might be necessary for the inhibition of neuronal apoptosis in slowly progressing neurodegenerative disorders, the use of autologous proteins is preferable to viral proteins in order to avoid potential immunological problems.

We have also applied an adenoviral vector coding for the neurotrophic factor, CNTF, to the cut nerve stump. Although the mechanisms of neuroprotection and antiapoptotic properties afforded by CNTF are less well-defined than those of XIAP, we determined whether this secreted neurotrophic factor might be more effective in RGC rescue than the intracellular XIAP. In the construct employed, the CNTF had an amino terminal fusion to the NGF leader sequence, permitting the recombinant protein to be secreted from the transduced cells. This has indeed been shown recently for the vector used in this study. ${ }^{29}$ The CNTF vector was efficient in rescuing RGCs after optic nerve axotomy and subsequent intraocular vector injection, which leads to transduction mainly of Müller glia cells and some amacrine and bipolar cells in the retina. ${ }^{35}$ After application on the nerve stump, however, Ad.CNTF administration did not rescue more ganglion cells than either the Ad.Xiap and Ad.p35 vectors, both of which code for intracellular proteins. This surprising result might either be due to the fact that CNTF acts only as a ligand for receptors on transduced cells or that not enough protein is secreted to be protective on larger cell numbers. It is interesting to note that in another paradigm of neuronal degeneration after axotomy (sciatic nerve lesion in newborn rats), intracellular anti-apoptotic proteins (NAIP, hIAP1 and hIAP2) were also as effective in cell rescue as the secreted neurotrophic factors BDNF and CNTF (Kato and Perrelet, personal communication).

As compared to our previous studies with the Ad.p35 and Ad.CrmA vector application, ${ }^{30}$ the administration protocol of the vectors was slightly modified in this study. In our previous work we applied the vectors to the nerve stump for only $5 \mathrm{~min}$, then removed the suspension and subsequently applied the tracer Fast Blue in order to label RGCs. Since the retinal ganglion cells in the present work have been prelabeled with the fluorescent tracer $\mathrm{Di}-\mathrm{I}$ by injection into the superior colliculi, it was not necessary to remove the vector suspension from the nerve stump. We found no difference in the number of RGCs surviving axotomy with either application mode for the Ad.p35 vector. In contrast to previous results, however, we clearly detected expression from the viral vectors 14 days after transduction of RGCs. RT-PCR analysis for the transgene expression was only semi-quantitative, since amplification of the internal reverse-transcription and amplification standard was carried out in reactions separately from the transgene's amplification, but we clearly detected a moderate decrease in the expression levels of all three transgenes. When comparing expression levels of XIAP, p35 and CNTF at 14 days after transduction to those at 7 days after transduction, we found a reduction to about $60 \%$. Either, the viral promoters (MCMV) of the adenoviral vectors were attenuated as has been demonstrated for HCMV, RSV and LTR promoters in cell culture and organotypical slices ${ }^{36,37}$ or some transduced RGCs sequentially degenerate despite the presence of the antiapoptotic proteins. Since we have shown that the viral vectors are taken up in different quantity by individual axons, only those cells which were transduced by multiple copies of the vectors might survive for the 14 day period post-lesion. However, even if the apoptotic cell death is only postponed in the axotomized neurons, this delay in apoptosis execution might offer the possibility for additional interventions in order to prevent cell death ultimately.

It has recently been shown that caspase 3 is constitutively expressed in the adult rat retina. The activation of the zymogen occurred frequently in the outer nuclear layer and, after axotomy, in retinal ganglion cells. ${ }^{38}$ Since a turnover of RGCs is impossible due to their long projection towards the superior colliculi, the expression of endogenous XIAP, as detected in unlesioned control retinae, may serve as a protective mechanism against sporadic activation of caspases. XIAP completely inhibits caspase 3 activity at equimolar ratios ${ }^{22}$ and is cleaved into fragments with lower inhibitory properties only if caspase 3 is present in molar excess. Overexpression of XIAP from the respective adenoviral vector rescued a substantial number of axotomized RGCs from apoptosis. Therefore, it is tempting to speculate that the RGCs surviving axotomy without any neuroprotective treatment do so because they express amounts of XIAP high enough to inhibit caspase mediated apoptotic cell death without the need to overexpress this protein by gene transfer. At present, however, we cannot quantify the relative amounts of neuroprotection deriving from either endogenous or ectopically expressed XIAP. Further studies will have to prove whether endogenous XIAP expression at various levels is sufficient to inhibit apoptosis in axotomized RGCs.

In summary, the work presented here clearly demonstrates that the $\mathrm{X}$-linked inhibitor of apoptosis (XIAP) is a useful factor for the inhibition of neuronal apoptosis after axonal lesion in the CNS. This is strengthened by recent results of others, who observed neuroprotection through XIAP overexpression in a model of transient ischemia. ${ }^{9}$ Together with the finding, that NAIP, hIAP-1 and -2 proteins were protective after peripheral motoneuron axotomy (Kato and Perrelet, pers. communication), IAP family members represent a promising group of anti-apoptotic factors with the potential for neuroprotective therapy in neurodegenerative disorders.

As the primary trigger for apoptosis induction in axotomized RGCs remains unknown to date, the delivery of proteins which specifically interact in different apoptotic pathways may shed light on the mechanisms whereby neurons degenerate after an axonal lesion. In addition, the application of vectors coding for proteins of the Bcl-2 family and the Bax-inhibitor ${ }^{39}$ will in the future help to define the 
participation of apoptosis induction via the mitochondrial pathway in these neurons in vivo.

\section{Material and Methods}

\section{Prelabeling of retinal ganglion cells and animal surgery}

To allow for accurate counting of RGCs, cells were retrogradely labeled with the fluorescent tracer $1,1^{\prime}$-dioctadecyl-3,3,3',3'-tetramethylindocarbocyanine perchlorate (Di-l; Molecular Probes Inc., Oregon, USA). The skull cartilage of rat pups (postnatal day 7) was opened dorsal of the lambda fissure under diethylether anesthesia, and both superior colliculi were instilled with $5 \%$ Di-I in dimethylformamide using a micropipette. Optic nerve surgery was performed with prelabeled animals at the age of 10 weeks. Briefly, prelabeled animals were anesthetized by intraperitoneal injection of chloral hydrate $(0.42 \mathrm{~g} / \mathrm{kg}$ bodyweight). After skin incision close to the superior orbital rim, the orbita was opened taking care to leave the supraorbital vein intact. Following subtotal resection of the lacrimal gland, the superior extraocular muscles were spread by means of a small retractor. The ON was exposed by longitudinal incision of the eye retractor muscle and the perineurium. ON transection was performed in a distance of approximately $2 \mathrm{~mm}$ from the posterior pole of the eye without damaging retinal blood supply. Animals with persistent retinal ischemia (verified fundoscopically) were not included in the study. Viral vectors were applied to the ocular side of the nerve stump by placing a drop of about $5 \mu \mathrm{l}$ into the opened dura. The viscosity of the solution used to store the virus (PBS/10\% glycerol) retained the solution in the cavity of the opened dura. Immediately after vector application, the wound was closed.

Application of vector into the optic layer of the superior colliculus was performed by stereotaxic injections: one injection was performed at bregma $-5.8 \mathrm{~mm}$, lateral $1 \mathrm{~mm}$, dorsoventral $4.2 \mathrm{~mm}$, a second injection was performed at bregma $-6.5 \mathrm{~mm}$, lateral $2.2 \mathrm{~mm}$, dorsoventral $4.2 \mathrm{~mm} .5 \times 10^{8}$ p.f.u. of Ad.lacZ in $5 \mu$ l were injected in $5 \mathrm{~min}$ in each injection.

\section{Quantification of surviving RGCs}

Fourteen days after ON transection, animals received an overdose of chloral hydrate, and the eyes were removed. The retinae were dissected, flat-mounted on gelatin-coated glass slides, and fixed for $20 \mathrm{~min}$ in 4\% paraformaldehyde (PFA) in PBS. RGCs were examined under the fluorescence microscope (Axioplan 2, Zeiss, Jena, Germany). The number of Di-I positive RGCs was determined by counting them in 12 distinct areas of $62500 \mu \mathrm{m}^{2}$ each (three areas per retinal quadrant at three different eccentricities of $1 / 6,1 / 2$ and $5 / 6$ of the retinal radius). Cell counts were performed according to a double blind protocol by two different investigators.

\section{Statistics}

Data are given as means \pm standard error of the mean (s.e.m.). Statistical significance was assessed using one-way ANOVA followed by Duncan test. Statistical analysis was performed using the absolute numbers of labeled RGCs. The number of cells surviving due to viral transduction was determined by subtracting the number of cells surviving without therapy from the values obtained with the different vector applications. Pairwise comparisons of groups was performed by a non-parametrical analysis of independent samples and MannWhitney-U-test.

\section{Vector construction}

Recombinant adenoviral vectors were constructed by homologous recombination in 293 cells according to standard procedures. ${ }^{40}$ The pBHGE3 backbone plasmid and the pMH4 shuttle plasmid were used, except for the Ad.CNTF, which was constructed in d1309 as described elsewhere. ${ }^{29}$ The pMH4 plasmid contains the short version (530 bp) of the murine cytomegalovirus (MCMV) promoter. Construction of the Ad.p35, Ad.dE1 and Ad.GFP has been described. ${ }^{30}$ Ad.Xiap was constructed as follows: the coding sequence of the rat Xiap cDNA was excised from the pGEX-RIAP3 plasmid by BamHI-Xhol digestion. The Xiap fragment was cloned into the respective siters of the pCDNA6myc-HIAP1 plasmid, from which the HIAP1 fragment has been removed. This results in a coding sequence for rat Xiap with an $\mathrm{N}$ terminal fusion of six copies of the myc epitope. By partial HindllIXhol digestion the insert was isolated, blunt-ended and cloned into the blunt-ended BamHI site of the $\mathrm{pMH} 4$ shuttle plasmid. Protein production from the Ad.Xiap vector was verified by Western blotting after transduction of MRC-5 fibroblasts and detection of an appropriate product in protein lysates with the anti myc antibody 9E10 (Santa Cruz, Heidelberg, Germany).

Recombinant virus was grown in suspension culture, purified and concentrated by two rounds of $\mathrm{CsCl}_{2}$ density gradient centrifugation and titers of transducing particles were determined by plaque assay. The amount of replication competent adenovirus (RCA) in the purified preparations was determined by a quantitative PCR approach, using primers as described. ${ }^{29}$ If RCA was detectable it was found to be at least six orders of magnitude below the titres for recombinant vectors. All vector preparations contained negligible endotoxin levels below $0.005 \mathrm{IE} / \mu \mathrm{l}$ as determined by the quantitative cromogenic LAL assay (BioWhittaker, Walkersville, USA).

\section{RNA preparation and RT-PCR}

Detection of transgene expression in transduced retinae was performed by RT-PCR. Total RNA was extracted from control retinae and from retinae transduced for either 7 or 14 days with the respective recombinant virus, using the RNeasy kit (Qiagen, Hilden, Germany). Two to three eyes per group were used and the RNA was pooled prior to analysis. The RNA was spectrophotometrically quantified. One $\mu \mathrm{g}$ of RNA was digested with DNase and then was reverse transcribed with a Xiap specific primer (5'-gag atg tag act gtg tgg cac cgt-3') or p35 specific primer (5'-cgg caa tat taa act ctg cac aaa ctc ttc- $\left.3^{\prime}\right)$ or a CNTF specific primer (5'-gta aga gta tgt att gcc tga tgg aag-3') using Superscript reverse transcriptase (Life Technologies, Karlsruhe, Germany). Each reaction also contained a G3PDH specific reverse transcription primer, $5^{\prime}$-cca cgg cca tca cgc cac agc ttt c- $3^{\prime}$. 10 pmoles of the Xiap, CNTF and p35 rt primers and 1 pmole of the G3PDH rt primer were used in the reverse transcriptions.

Nested PCRs were performed with the following primers: Xiap, 1st amplification: (5'-gaa atg gag agc ttg ggc ga- $\left.3^{\prime}\right)$ and ( $5^{\prime}$-ttg ggg ata ttc tcc tgt gtc ttc- $\left.3^{\prime}\right)$, 2nd amplification: (5'-cat gga gca aaa gct cat ttc t- $\left.3^{\prime}\right)$ and ( $5^{\prime}$-tct atc tac tgc cgc gtg aca-3'); CNTF, 1st amplification: (5'-tgt tgt tct aca ctc tga tca ct- $\left.3^{\prime}\right)$ and ( $5^{\prime}$-tga aat gca ctc tct ggt c- $\left.3^{\prime}\right)$, 2nd amplification ( $5^{\prime}$-tca cgt cgt ttt tga tcg- $\left.3^{\prime}\right)$ and (5'-ctt gga agg tac ggt aag c-3'), p35, 1st amplification: (5'-tca aat gga tgg att cca cg-3') and (5'-cca tca aac tgc aca ctg tc- $\left.3^{\prime}\right)$, 2nd amplification: (5'-tga aca cta ttc ggt aag ttg c-3') and (5'-gac gtc gta ctc gta aag tcc-3'); G3PDH mRNA was amplified with primers $5^{\prime}$-cacggcaagttcaatg- $3^{\prime}$ and $5^{\prime}$-tctgagtgg- 
cagtgatg-3'. Cycling was performed with vent exo- polymerase (New England Biolabs, Schwalbach, Germany).

\section{Histochemical detection of $\beta$-galactosidase activity and immunohistochemistry}

Five days after axotomy and transduction with the Ad.lacZ vector, complete eyes were prepared. Specimens were fixed for $30 \mathrm{~min}$ in $1 \%$ PFA/solution 1 (100 mM K ${ }_{2} \mathrm{HPO} 4,5 \mathrm{mM}$ EDTA, $2 \mathrm{mM} \mathrm{MgCl}_{2}, 0.02 \%$ NP40) and then rinsed three times in excessive volumes of solution 1 and stained overnight at $37^{\circ} \mathrm{C}$ in solution 2 (solution $1+10 \mathrm{mM}$ $\mathrm{K}_{3} \mathrm{Fe}(\mathrm{CN})_{6} \mathrm{FC}, 10 \mathrm{mM} \mathrm{K}{ }_{4} \mathrm{Fe}(\mathrm{CN})_{6} \mathrm{FC}, 750 \mathrm{mg} / \mathrm{ml} \mathrm{X}$-gal). After PBS wash, the eyes were embedded in TissueTek and frozen. Fourteen $\mu \mathrm{m}$ sections were prepared, postfixed in 4\% PFA and counterstained with DAPI. For immunofluorescent detection, a polyclonal antibody specific for $\beta$-galactosidase was used (MoBiTek, Goettingen, Germany) at a dilution of $1: 50$ to stain $16 \mu \mathrm{m}$ cryostat sections mounted to slides. Fluorescent detection was performed by using a Cy2 coupled antirabbit-IgG on a Zeiss Axiophot 2 microscope and recorded by a CCD Kamera (Zeiss, Goettingen, Germany). Expression of XIAP was detected by using a polyclonal anti-XIAP antibody $(1: 100)$. Fluorescence from the Cy3 coupled secondary antibody was recorded either as above or on a confocal laser scanning microscope (LSN 510, Zeiss, Goettingen, Germany).

\section{Acknowledgements}

We thank Liane Meyn for excellent technical assistance and C. Gravel for the Ad.CNTF vector. We are grateful to Ricarda Diem for providing fluorogold labeled retinal sections. This work was supported by a grant from the Federal Ministry of Education, Science, Research and Technology (Fö 01KS9602) and the Interdisciplinary Center of Clinical Research (IZKF) Tübingen.

\section{References}

1. Bergeron L and Yuan J (1998) Sealing one's fate: control of cell death in neurons. Curr. Opin. Neurobiol. 8: 55-63

2. Jacobson MD, Weil M and Raff MC (1997) Programmed cell death in animal development. Cell 88: 347-354

3. Iwahashi $H$, Eguchi $Y$, Yasuhara N, Hanafusa T, Matsuzawa $Y$ and Tsujimoto $Y$ (1997) Synergistic anti-apoptotic activity between Bcl-2 and SMN implicated in spinal muscular atrophy. Nature 390: 413-417

4. Kim TW, Pettingell WH, Jung YK, Kovacs DM and Tanzi R (1997) Alternative cleavage of Alzheimer-associated presenilins during apoptosis by a caspase-3 family protease. Science $277: 373-376$

5. Roperch JP, Alvaro V, Prieur S, Tuynder M, Nemani M, Lethrosne F, Piouffre L, Gendron MC, Israeli D, Dausset J, Oren M, Amson R and Telerman A (1998) Inhibition of presenilin 1 expression is promoted by p53 and p21(WAF-1) and results in apoptosis and tumor suppression. Nature Med. 4: 835-838

6. Gervais FG, Xu D, Robertson GS, Vaillancourt JP, Zhu Y, Huang J, LeBlanc A Smith D, Rigby M, Shearman MS, Clarke EE, Zheng H, Van-Der-Ploeg LH, Ruffolo SC, Thornberry NA, Xanthoudakis S, Zamboni RJ, Ros S and Nicholson DW. (1999) Involvement of caspases in proteolytic cleavage of Alzheimer's amyloid- $\beta$ precurser protein and mayloidogenic $\mathrm{A} \beta$ peptide formation. Cell 97: 395-406

7. Namura S, Zhu J, Fink K, Endres M, Srinivasan A, Tomaselli KJ, Yuan J and Moskowitz MA (1998) Activation and cleavage of caspase-3 in apoptosis induced by experimental cerebral ischemia. J. Neurosci. 18: 3659-3668

8. Guglielmo MA, Chan PT, Cortez S, Stopa EG, McMillan P, Johanson CE, Epstein $M$ and Doberstein CE (1998) The temporal profile and morphologic features of neuronal death in human stroke resemble those observed in experimental forebrain ischemia: the potential role of apoptosis. Neurol. Res. 20: 283-296
9. Xu D, Bureau Y, McIntyre DC, Nicholson DW, Liston P, Zhu Y, Fong WG, Crocker SJ, Korneluk RG and Robertson GS (1999) Attenuation of ischemia-induced cellular and behavioral deficits by $\mathrm{X}$ chromosome-linked inhibitor of apoptosis protein overexpression in the rat hippocampus. J. Neurosci. 19: 5026-5033

10. Conti AC, Raghupathi R, TrojanowskiJQ and McIntosh-TK (1998) Experimental brain injury induces regionally distinct apoptosis during the acute and delayed post-traumatic period. J. Neurosci. 18: 5663-5672

11. Cohen GM (1997) Caspases: the executioners of apoptosis. Biochem. J. 326: $1-16$

12. Nicholson DW and Thornberry NA (1997) Caspases: killer proteases. Trends. Biochem. Sci. 8: 299-306

13. Zhou Q, Krebs JF, Snipas SJ, Price A, Alnemri ES, Tomaselli KJ and Salvesen GS (1998) Interaction of the baculovirus anti-apoptotic protein p35 with caspases. Specificity, kinetics, and characterization of the caspase/p35 complex. Biochemistry 37: 10757-10765

14. Clem RJ and Miller LK (1994) Prevention of apoptosis by a baculovirus gene during infection of insect cells. Mol. Cell. Biol. 14: 5212-5222

15. Zhou Q, Snipas S, Orth K, Muzio M and Dixit VM (1997) Target protease specificity of the viral serpin CrmA. Analysis of five caspases. J. Biol. Chem. 272: $7797-7800$

16. Thome M, Schneider P, Hofmann K, Fickenscher H, MeinlE, Neipel F, Mattmann C, Burns K, Bodmer JL, Schroter M, Scaffidi C, Krammer PH, Peter ME and Tschopp J (1997) Viral FLICE-inhibitory proteins (FLIPs) prevent apoptosis induced by death receptors. Nature 386: $517-521$

17. Deveraux QL, Takahashi R, Salvesen GS and Reed JC (1997) X-linked IAP is a direct inhibitor of cell death proteases. Nature 388: $300-304$

18. Roy N, Mahadevan MS, McLean M, Shutler G, Yaraghi Z, Farahani R, Baird S, Besner-Johnson A, Lefebvre C, Kang X, Sabih M, Aubry H, Tamai K, Guan X, loannou P, Crawford TO, de Jong PJ, Surh L, Ikeda JE, Korneluk RG and MacKenzie A (1995) The gene for neuronal apoptosis inhibitory protein is partially deleted in individuals with spinal muscular atrophy. Cell 80: 167-178

19. Deveraux QL, Roy N, Stennicke HR, Van-Arsdale T, Zhou Q, Srinivasula SM, Alnemri ES, Salvesen GS and Reed JC (1998) IAPs block apoptotic events induced by caspase-8 and cytochrome c by direct inhibition of distinct caspases. EMBO J. 17: 2215-2223

20. Tamm I, Wang Y, Sausville E, Scudiero DA, Vigna N, Oltersdorf T and Reed JC (1998) IAP-family protein survivin inhibits caspase activity and apoptosis induced by Fas (CD95), Bax, caspases, and anticancer drugs. Cancer Res. 58: $5315-5320$

21. Tschopp J, Irmler M, Thome M (1998) Inhibition of Fas death signals by FLIPs. Curr. Opin. Immunol. 10: 552-558

22. Deveraux QL, Leo E, Stennicke HR, Welsh K, Salvesen GS and Reed JC (1999) Cleavage of human inhibitor of apoptosis protein XIAP results in fragments with distinct specificities for caspases. EMBO J. 18: $5242-5251$

23. Garcia-Valenzuela E, Gorczyca W, Darzynkiewicz Z and Sharma SC (1994) Apoptosis in adult retinal ganglion cells after axotomy. J. Neurobiol. 25: 431-438

24. Rabacchi SA, Bonfanti L, Liu XH and Maffei L (1994) Apoptotic cell death induced by optic nerve lesion in the neonatal rat. J. Neurosci. 14: 5292-5301

25. Isenmann S, Wahl C, Krajewski S, Reed JC and Bähr M (1997) Up-regulation of Bax protein in degenerating retinal ganglion cells precedes apoptic cell death after optic nerve lesion in the rat. Eur. J. Neurosci. 9: 1763-1772

26. Klöcker N, Cellerino A and Bähr M (1998) Free radical scavenging and inhibition of nitric oxide synthase potentiates the neurotrophic effects of brain-derived neurotrophic factor on axotomized retinal ganglion cells in vivo. J. Neurosci. 18 : $1038-1046$

27. Kermer P, Klöcker N, Labes M and Bähr M (1998) Inhibition of CPP32-like proteases rescues axotomized retinal ganglion cells from secondary cell death in vivo. J. Neurosci. 18: 4656-4662

28. Cayouette M and Gravel C (1996) Adenovirus-mediated gene transfer to retinal ganglion cells. Inv. Ophtal. Vis. Sci. 37: 2022-2028

29. Gravel C, Gotz R, Lorrain A and Sendtner M (1997) Adenoviral gene transfer of ciliary neurotrophic factor and brain-derived neurotrophic factor leads to longterm survival of axotomized motor neurons. Nat. Med. 3: 765-770

30. Kügler S, Klöcker N, Kermer P, Isenmann S and Bähr M (1999) Transduction of axotomized retinal ganglion cells by adenoviral vector administration at the optic nerve stump: an in vivo model system for the inhibition of neuronal apoptotic cell death. Gene Ther. 6: 1759-1767

31. Barinaga M. (1998) Stroke-damaged neurons may commit cellular suicide. Science 281: 1302-1303 
32. Roy N, Deveraux QL, Takashashi R, Salvesen GS and Reed JC (1997) The CIAP-1 and c-IAP-2 proteins are direct inhibitors of specific caspases. EMBO J. 16 6914-6925

33. Chu ZL, McKinsey TA, Liu L, Gentry JJ, Malim MH and Ballard DW (1997) Suppression of tumor necrosis factor-induced cell death by inhibitor of apoptosis c-IAP2 is under NF-kappaB control. Proc. Natl. Acad. Sci. USA 94: 1005710062

34. Stehlik C, de Martin R, Kumabashiri I, Schmid JA, Binder BR and Lipp J (1998) Nuclear factor (NF)-kappaB-regulated X-chromosome-linked iap gene expressin protects endothelial cells from tumor necrosis factor alpha-induced apoptosis. J. Exp. Med. 188: 211-216

35. Weise J, Isenmann S, Klöcker N, Kügler S, Gravel C and Bähr M. Adenovirus mediated expression of ciliary neurotrophic factor (CNTF) rescues axotomized rat retinal ganglion cells but does not support axonal regeneration in vivo. Neurobiol. Dis. in press
36. Reich N, Pine R, Levy D and Darnell JE Jr (1988) Transcription of interferonstimulated genes is induced by adenovirus particles but is suppressed by $\mathrm{E} 1 \mathrm{~A}$ gene products. J. Virol. 62: 114-119

37. Harms JS and Splitter GA (1995) Interferon-gamma inhibits transgene expression driven by SV40 or CMV promoters but augments expression driven by the mammalian MHC1 promoter. Hum. Gene Ther. 6: 1291-1297

38. Kermer P, Klöcker N, Labes M, Thomsen S, Srinivasan A and Bähr M (1999) Activation of caspase-3 in axotomized rat retinal ganglion cells in vivo. FEBS Lett. 453: $361-364$

39. Xu Q and Reed JC (1998) Bax inhibitor-1, a mammalian apoptosis suppressor identified by functional screening in yeast. Mol. Cell. 1: $337-346$

40. Bett AJ, Haddara W, Prevec $L$ and Graham FL (1994) An efficient and flexible system for construction of adenovirus vectors with insertions or deletions in early regions 1 and 3. Proc. Natl. Acad. Sci. USA 91: 8802-8806 